“C2019 IEEE. Personal use of this material is permitted. Permission from IEEE must be obtained for all other uses, in any current or future media, including reprinting/republishing this material for advertising or promotional purposes, creating new collective works, for resale or redistribution to servers or lists, or reuse of any copyrighted component of this work in other works." 


\title{
Design and Experimental Demonstration of Compact Polarization Reconfigurable Antennas
}

\author{
Ming-Chun Tang ${ }^{1}$, Zhentian $\mathrm{Wu}^{1}$, Dajiang $\mathrm{Li}^{1}$, Richard W. Ziolkowski ${ }^{2}$ \\ ${ }^{1}$ College of Microelectronics and Communication Engineering, Chongqing University, Chongqing, China, \\ tangmingchun@cqu.edu.cn \\ ${ }^{2}$ Global Big Data Technologies Centre, University of Technology Sydney, Ultimo NSW 2007, Australia \\ Richard.Ziolkowski@uts.adu.au
}

\begin{abstract}
Two compact polarization reconfigurable antennas are presented. The first is an electrically small, low-profile, planar, Huygens source antenna with four reconfigurable polarization states. It has very useful realized gain values in its broadside direction, symmetric radiation patterns, and low back radiation in all four polarization states. The second is a compact, wideband, planar tri-polarization reconfigurable filtenna. It exhibits a wide impedance bandwidth, stable realized gain, and good out-of-band rejection performance for all three of its reconfigurable states. Both of these compact polarization reconfigurable antennas were fabricated and tested. The measured results, in good agreement with their simulated values, demonstrate their efficacy to reconfigure their polarization states.
\end{abstract}

\section{INTRODUCTION}

Near-field resonant parasitic (NFRP) technologies have been used to realize electrically small Huygens antennas (ESHAs) [1-6]. These antennas have been studied in recent years because they can achieve high directivity and broadside radiation patterns without significantly increasing their size and profile. Consequently, they provide significant advantages for many space-limited wireless communication platforms. On the other hand, filtennas combine filters and antennas into a single module [7-9] to reduce the interconnection lengths and losses between the filter and the radiating elements. Moreover, they can be designed to have excellent frequency selectivity and out-of-band rejection performance. Thus, they facilitate more compact and lower cost RF front-ends.

Nevertheless, these ESHA and filtenna designs and their successful realizations would be more attractive for mobile wireless systems if they were versatile. For example, if they were polarization reconfigurable, they would have many advantages. These include mitigating polarization mismatch, reducing channel interference, improving system capacity, and realizing multiple transmission channels for frequency reuse [10]. Therefore, it is highly desirable to empower ESHAs and filtennas with polarization reconfigurability.

In this paper, two compact polarization reconfigurable antennas are presented. The first is a four-state, reconfigurable ESHA [11]. This very compact design incorporates both electric and magnetic NFRP elements and a reconfigurable driven element. Reconfigurability is achieved with only six p-i$\mathrm{n}$ (PIN) diodes. By manipulating their ON/OFF states, this antenna dynamically achieves four polarization states which include two orthogonal linear (LP) and two circular polarization (LHCP and RHCP) states. The measured results demonstrate that it is electrically small $(k a=0.944)$ and low profile $\left(0.0449 \lambda_{0}\right)$ at $1.5 \mathrm{GHz}$. The second is a compact, wideband, planar three-state reconfigurable filtenna [12]. Reconfigurability is achieved by integrating four pairs of PIN diodes into its feed network to realize three polarization states, i.e., one LP and two CP (LHCP and RHCP) states.

\section{RECONFIGURABLE ESHA}

\section{A. Design}

Fig. 1 shows the optimized reconfigurable ESHA configuration. Electric and magnetic NFRP elements are combined with a coax-fed cross-dipole antenna. The ESHA is comprised of three cylindrical substrate layers, labeled as Layer_1, Layer_2 and Layer_3, respectively. All three substrate layers have the same radius, $\mathrm{R} 1=30.0 \mathrm{~mm}$, and were selected to be Taconic TLY-5. However, each layer has a different thickness, i.e., $\mathrm{h} 1=\mathrm{h} 2=0.25 \mathrm{~mm}$, and $\mathrm{h} 3=1.58 \mathrm{~mm}$.

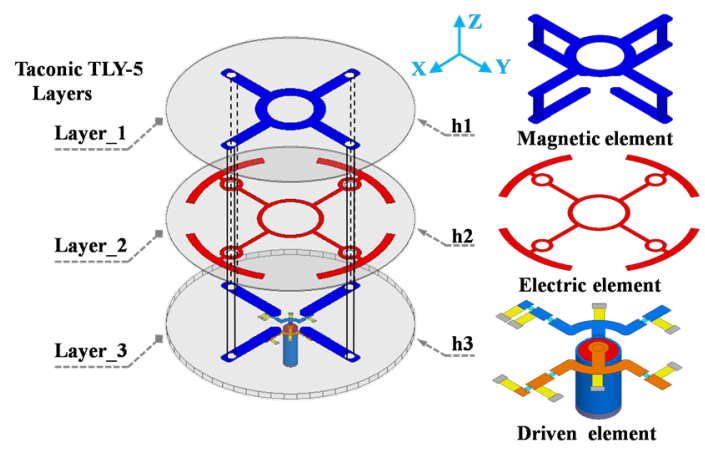

Fig. 1. Polarization reconfigurable ESHA configuration.

The magnetic NFRP elements are two same-sized, orthogonally-oriented capacitively loaded loops (CLLs). One CLL is oriented along the $y$-axis, and the other CLL is oriented along the $x$-axis. The upper surface of the magnetic element is located on the upper surface of Layer_1 and the lower surface of this element is located on the upper surface of Layer_3. They are connected by four copper cylindrical columns. Similarly, the electric NFRP elements are two same-sized Egyptian axe dipoles (EADs). They are located on the upper surface of Layer_2. One is oriented along the $y$-axis, and the other is oriented along the $x$-axis. The coax-fed cross-dipole driven element lies on the lower surface of Layer_3. 


\section{B. Simulated and measured results}

The fabricated and assembled four-state, reconfigurable ESHA is shown in Fig. 2(a). The reconfigurable driven element is shown in Fig. 2(b). Six MACOM MA4GP907 PIN diodes and eight Coilcraft inductors were employed to achieve the reconfigurability. The simulated and measured resonance frequency, fractional bandwidth (FBW), axial ratio (AR) FBW, front-to-back ratio (FTBR), realized gain, and radiation efficiency (RE) results in all four states are summarized in Table I for easy reference.

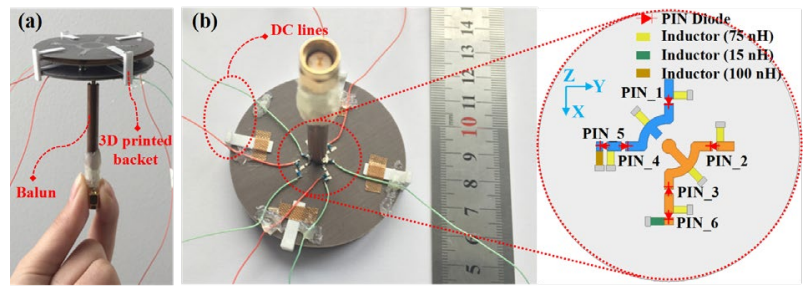

Fig. 2. Fabricated prototype of the reconfigurable ESHA. (a) Side view. (b) Bottom view with inset of the coax-fed PIN-diode augmented cross-dipole element.

TABLE I

Simulated AND MEASUREd Results OF All FOUR Polarization StateS OF THE ReConfigurable ESHA. The MEASURED (Simulated) Results ARE IN THE SHADED (CLEAR) BOXES.

\begin{tabular}{|c|c|c|c|c|}
\hline State & $x$-LP & $y$-LP & LHCP & RHCP \\
\hline \multirow{2}{*}{$\begin{array}{l}\text { Resonance frequency } \\
(\mathrm{GHz})\end{array}$} & 1.508 & 1.509 & 1.501 & 1.501 \\
\hline & 1.507 & 1.512 & 1.501 & 1.5 \\
\hline \multirow{2}{*}{$\left|\mathrm{S}_{11}\right|_{\min }(\mathrm{dB})$} & -41.6 & -30.0 & -19.5 & -16.5 \\
\hline & -12.4 & -12.0 & -16.5 & -13.0 \\
\hline \multirow{2}{*}{ FBW (\%) } & 0.86 & 0.86 & 1.33 & 1.13 \\
\hline & 0.92 & 1.1 & 1.33 & 1.06 \\
\hline \multirow{2}{*}{$\begin{array}{l}\text { Axial ratio FBW (\%) } \\
(\mathrm{AR} \leq 3 \mathrm{~dB})\end{array}$} & & & 0.34 & 0.36 \\
\hline & & & 0.19 & 0.19 \\
\hline \multirow{2}{*}{ FTBR (dB) } & 11.2 & 11.0 & 15.2 & 15.2 \\
\hline & 10.7 & 9.9 & 11.4 & 12.5 \\
\hline \multirow{2}{*}{ Realized gain $(\mathrm{dBi})$} & 3.51 & 3.54 & 3.13 & 2.99 \\
\hline & 3.03 & 2.97 & 2.82 & 2.74 \\
\hline \multirow{2}{*}{$\begin{array}{l}\text { Radiation efficiency } \\
\text { RE (\%) }\end{array}$} & 77.9 & 78.9 & 71.3 & 69.8 \\
\hline & 68.2 & 67.5 & 67.1 & 65.9 \\
\hline
\end{tabular}

\section{COMPACT RECONFIGURABLE FILTENNA}

\section{A. Design}

Fig. 3 illustrates the reconfigurable filtenna design. It consists of three square substrate layers which are labeled as Layer_1, Layer_2 and Layer_3, respectively. All of three substrates are Rogers RT/Duroid 5880 with a permittivity of $\varepsilon_{r}$ $=2.2$, a thickness of $0.787 \mathrm{~mm}$, and a size of $L_{l} \times W_{l}=120 \times$ $120 \mathrm{~mm}^{2}$. An air gap is introduced between Layer_1 and Layer_2. It enhances the antenna bandwidth and gain. With reference to the Fig. 3(a), a square parasitic patch is fabricated on the upper surface of Layer_1. The driven patch is fabricated on the upper surface of Layer_2.

A reconfigurable feed network was developed to realize the polarization reconfigurability amongst the one LP and two CP states. It is composed of a Wilkinson power divider, a set of phase-shift strips, and three coupling stubs. The Wilkinson power divider (green) and the phase-shift strips (blue) are all mounted on the upper surface of Layer_3. The three coupling stubs (magenta), labeled as Stub_1, Stub_2 and Stub_3, have the same size and are located on the lower surface of Layer_2. The coupling stubs and the phase-shift strips are connected by three metal posts (yellow). In Fig. 3(b), eight Infineon PIN diodes (BAR64-02V) in SC79 packages and six $95 \mathrm{nH}$ inductors in 0603 packages are integrated into the feed network. The reconfigurable filtenna was fabricated, assembled, and measured shown in Fig. 3(c).

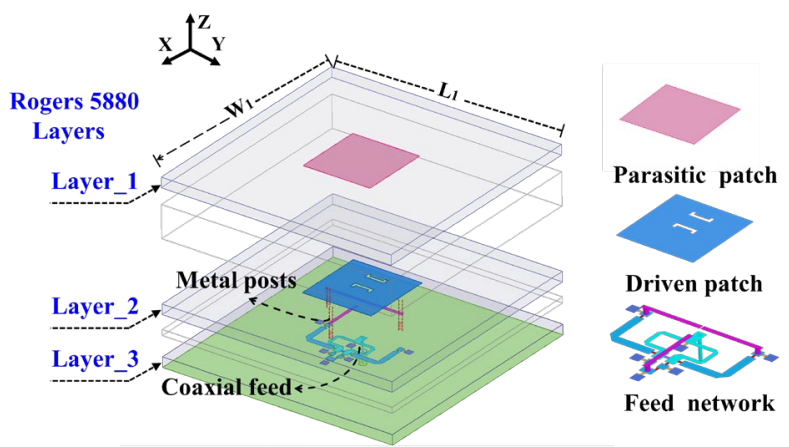

(a)

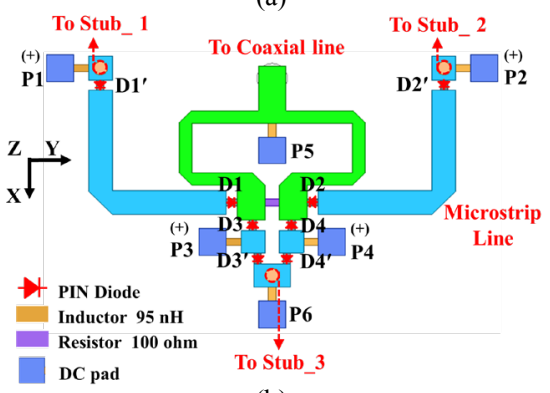

(b)

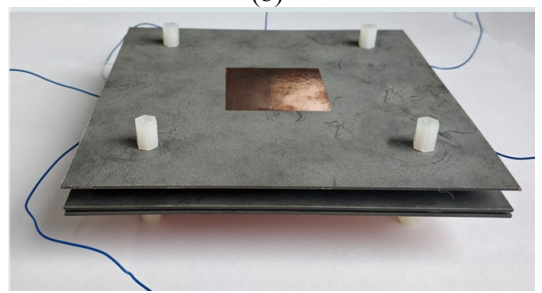

(c)

Fig. 3. Polarization reconfigurable filtenna. (a) 3-D isometric view. (b) Feed network and the locations of the PIN diodes. (c) 3-D isometric view of assembled filtenna.

\section{B. Simulated and measured results}

As is shown in Fig. 4, the reconfigurable filtenna was fabricated, assembled, and measured. The measured results in Fig. 6 are in good agreement with their simulated values. They indicate that the filtenna exhibits a wide $15.6 \% \mathrm{FBW}$ centered at $2.9 \mathrm{GHz}$ with a flat broadside average realized gain, $7.7 \pm$ $0.5 \mathrm{dBi}$. The corresponding simulated RE values are all over $75 \%$ within this operational band. Note that the effective bandwidth refers to the overlap of the bandwidths of all three states. Moreover, three realized gain zeroes (one in the lower band and two in the upper band) were produced. As a consequence, the filtenna exhibits a good out-of-band rejection 
performance in all of its three polarization states. The simulated and measured FBW, realized gain, and AR FBW results in all three states are summarized in Table II for easy reference.

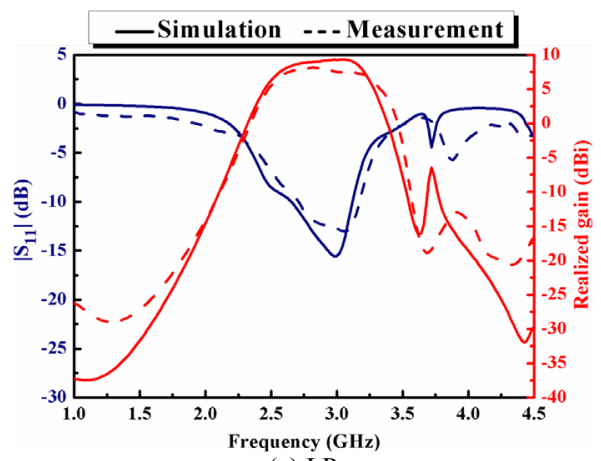

(a) LP

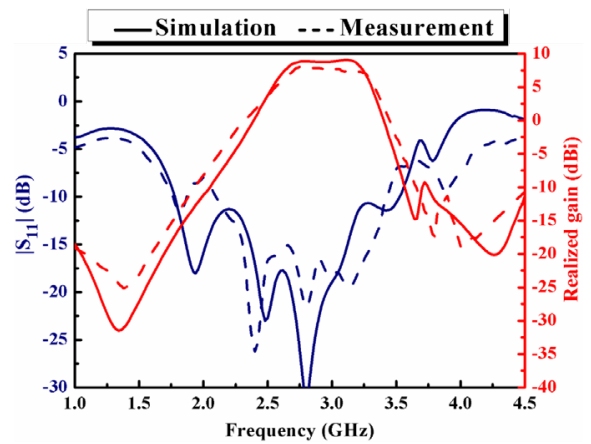

(b) LHCP

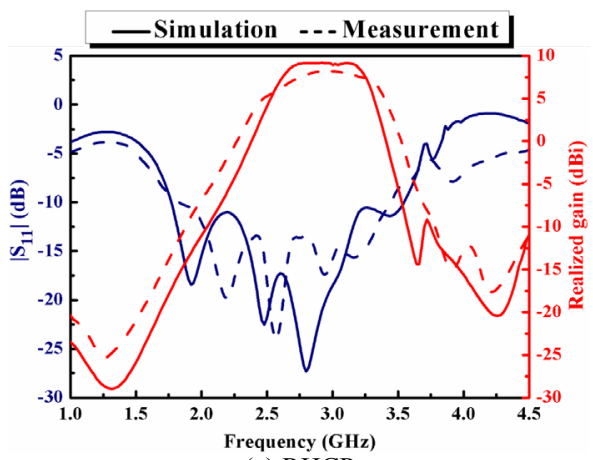

(c) RHCP

Fig. 4. Simulated and measured reflection coefficient, i.e., $\left|S_{11}\right|$, and realized gain values of the filtenna in its three polarization states.

TABLE II

Simulated and Measured Results for The POLARIZATION Reconfigurable Filtenna in All Polarization States. The Measured (Simulated) Results ARE IN THE SHADEd (Clear) BoXes

\begin{tabular}{|l|l|l|l|}
\hline State & LP & LHCP & RHCP \\
\hline \multirow{2}{*}{$\begin{array}{l}\text { Impedance FBW (\%) } \\
\left(\left|\mathrm{S}_{11}\right| \leq-10 \mathrm{~dB}\right)\end{array}$} & 16.3 & 66.4 & 67 \\
\cline { 2 - 4 } $\begin{array}{l}\text { Average realized gain } \\
(\mathrm{dBi})\end{array}$ & 15.6 & 62.6 & 62.6 \\
\cline { 2 - 4 } & 7.8 & 8.6 & 8.6 \\
\hline \multirow{2}{*}{$\begin{array}{l}\text { AR FBW (\%) } \\
(\mathrm{AR} \leq 3 \mathrm{~dB})\end{array}$} & & 7.7 & 7.7 \\
\hline
\end{tabular}

\section{CONCLUSION}

Two compact polarization reconfigurable antennas were presented. The first system was a low-profile, planar, electrically small, Huygens antenna with four reconfigurable polarization states. Different from previously successful Huygens dipole NFRP elements, the reported quad-polarized reconfigurable design was achieved through the development of a novel, reconfigurable driven element implemented with six PIN diodes. The second system was a wideband, planar filtenna with three reconfigurable polarization states, each having good out-of-band rejection performance. It has a compact size: $0.48 \lambda_{0} \times 0.45 \lambda_{0}$, and is low profile: height $=$ $0.096 \lambda_{0}$. The measured results of prototypes of each system were demonstrated to be in good agreement with their simulated values.

\section{REFERENCES}

[1] R. W. Ziolkowski, "Low profile, broadside radiating, electrically small Huygens source antennas," IEEE Access, vol. 3, pp. 2644-2651, 2015

[2] M.-C. Tang, H. Wang, and R. W. Ziolkowski, "Design and testing of simple, electrically small, low-profile, Huygens source antennas with broadside radiation performance," IEEE Trans. Antennas Propag., vol.64, pp. 4607-4617, November 2016.

[3] M.-C. Tang, T. Shi, and R. W. Ziolkowski, "A study of $28 \mathrm{GHz}$, planar, multi-layered, electrically small, broadside radiating, Huygens source antennas," IEEE Trans. Antennas Propag., in the Special Issue on "Antennas and Propagation Aspects of 5G Communications", vol. 65, pp. 6345-6354, December 2017.

[4] M.-C. Tang, B. Zhou, and R. W. Ziolkowski, "Low-profile, electrically small, Huygens source antenna with pattern-reconfigurability that covers the entire azimuthal plane," IEEE Trans. Antennas Propag., vol.65, pp. 1063-1072, March 2017.

[5] M.-C. Tang, T. Shi, and R. W. Ziolkowski, "Electrically small, broadside radiating Huygens source antenna augmented with internal non-Foster elements to increase its bandwidth," IEEE Antennas Wirel. Propag. Lett., vol.16, pp. 712-715, 2017.

[6] M.-C. Tang, Z. Wu, T. Shi, H. Zeng, W. Lin, and R. W. Ziolkowski, "Dual-linearly-polarized, electrically small, low-profile, broadside radiating, Huygens dipole antenna," IEEE Trans. Antennas Propag., vol.66, pp. 3877-3885, August 2017.

[7] M.-C. Tang, Y. Chen, and R. W. Ziolkowski, "Experimentally validated, planar, wideband, electrically small, monopole filtennas based on capacitively loaded loop resonators," IEEE Trans. Antennas Propag., vol. 64, pp. 3353-3360, August 2016.

[8] F. Farzami, S. Khaledian, B. Smida, and D. Erricolo, "Reconfigurable linear/circular polarization rectangular waveguide filtenna," IEEE Trans. Antennas Propag., vol. 66, pp. 9-15, January 2018.

[9] K.-Z. Hu, M.-C. Tang, M. Li, and R. W. Ziolkowski, "Compact, lowprofile, bandwidth-enhanced substrate integrated waveguide filtenna," IEEE Antennas Wireless Propag. Lett., vol. 17, pp. 1552-1556, 2018.

[10] F. Wu and K. M. Luk, "Single-port reconfigurable magneto-electric dipole antenna with quad-polarization diversity," IEEE Trans. Antennas Propag., vol. 65, pp. 2289-2296, May 2017.

[11] M.-C. Tang, Z. Wu, T. Shi, and R. W. Ziolkowski, "Electrically small, low-profile, planar, Huygens dipole antenna with quad-polarization diversity," IEEE Trans. Antennas Propag., early access, 2018.

[12] M.-C. Tang, D. Li, X. Chen, Y. Wang, K. Hu, and R. W. Ziolkowski, "Compact, wideband, planar filtenna with reconfigurable tri-polarization diversity," IEEE Trans. Antennas Propag., under review. 